\title{
Resiliência em estudantes do Ensino Médio
}

\author{
Lidiane Peltz \\ Maria da Graça Moraes \\ Mary Sandra Carlotto
}

\begin{abstract}
Resumo
O objetivo do estudo foi avaliar a resiliência em estudantes do Ensino Médio e sua associação com variáveis sociodemográficas e contribuição da escola em seu desenvolvimento pessoal. A amostra foi composta de 140 alunos de uma Escola Estadual de uma cidade da região metropolitana de Porto Alegre. Foram utilizados, como instrumentos de pesquisa, um questionário para levantamento de variáveis demográficas e sociais e a Escala de Resiliência, adaptada por Pesce, Assis, Avanci, Santos, Malaquias e Carvalhaes (2005), que constitui-se de três dimensões: Independência e determinação; Resolução de ações e valores; Autoconfiança e Capacidade de adaptação a situações. Resultados indicam associação negativa entre renda familiar e a dimensão de resiliência de Independência e determinação. Associação positiva foi identificada entre a percepção de que a escola contribui para o desenvolvimento pessoal e a dimensão de Resolução de ações e valores.
\end{abstract}

Palavras-chave: Resiliência, estudantes, ensino médio.

\section{Resilience of High School students}

\begin{abstract}
In this study we aim at evaluating the resilience of students in High School We are interested in assessing the association of student's resilience with socio-demographic variables and the school contribution to the students' personal development. The sample was composed by 140 students from a public school of () Porto Alegre's metropolitan area. The instrument used was a questionnaire survey of demographic and social variables and the Resilience Scale adapted by Pesce, Assis, Avanci, Santos, Malaquias and Carvalhaes (2005) .It consists of three dimensions: Independence and determination, Resolution of actions and values, Self-confidence and Capacity to adapt to situations. Results showed the negative association between family income and dimension of resilience of Independence and determination. Positive association was identified between the perception that the school contributes to the personal development and the dimension of Resolution of actions and values.
\end{abstract}

Keywords: Resilience, students, secondary education.

\section{Resiliencia en estudiantes de enseñanza secundaria}

\section{Resumen}

El objetivo del estudio fue evaluar la resiliencia en estudiantes de Enseñanza Secundaria y su asociación con variables socio-demográficas y la contribución de la escuela en su desarrollo personal. La muestra fue de 140 alumnos de una Escuela Estatal en una ciudad de la región metropolitana de Porto Alegre. Se utilizaron como instrumentos de investigación, un cuestionario para levantar variables demográficas y sociales y la Escala de Resiliencia, adaptada por Pesce, Assis, Avanci, Santos, Malaquias e Carvalhaes (2005), que se constituye de tres dimensiones: Independencia y determinación; Resolución de acciones y valores; Autoconfianza y Capacidad de adaptación a situaciones. Resultados indican la asociación negativa entre los ingresos familiares y la dimensión de resiliencia de Independencia y determinación. Asociación positiva fue identificada entre la percepción de que la escuela contribuye para el desarrollo personal y la dimensión de Resolución de acciones y valores. Palabras clave: Resiliencia, estudiantes, educación secundaria. 


\section{Introdução}

Já há algum tempo, a ciência tem-se interrogado sobre por que determinadas pessoas têm a capacidade de superar as piores situações, enquanto outras ficam comprometidas nas malhas da infelicidade e da angústia. As experiências e estudos feitos têm mostrado algumas explicações científicas sobre esse fato. A Biologia defende o ponto de vista de que cada ser humano é dotado de um potencial genético que o faz ser mais resistente que outros. A Psicologia, por sua vez, dá realce e importância às relações familiares, sobretudo na infância, que construirá nesse indivíduo a capacidade de suportar certas crises e de superá-las. A Sociologia vai fazer referência à influência do entorno, da cultura e das tradições como construtores dessa capacidade do indivíduo de suplantar as adversidades. Já a Teologia traz um aporte diferente pela própria subjetividade transcendente, uma visão outra da condição humana e da necessidade do sofrimento como fator de evolução espiritual (Vasconcelos, 2005). Essas experiências e estudos têm introduzido o conceito de resiliência.

Segundo Yunes (2003), na língua portuguesa, a palavra resiliência, aplicada às ciências sociais e humanas, vem sendo utilizada há poucos anos. No Brasil, seu uso ainda se restringe a um limitado grupo de pessoas e a alguns círculos acadêmicos. Muitos profissionais da área da Psicologia, da Sociologia ou da Educação ainda não tiveram contato com a palavra e desconhecem seu uso e aplicação em qualquer das áreas da ciência. No entanto, profissionais do campo da Engenharia, Ecologia, Física e Odontologia utilizam o termo para se referir à resistência de materiais. $O$ conceito de resiliência tem sua origem na Física e na Engenharia para indicar a propriedade pela qual a energia armazenada em um corpo deformado é devolvida quando cessa a tensão causadora de uma deformação elástica (Aznar-Farias \& Oliveira-Monteiro, 2006). Transportada para as Ciências Sociais, adquire um novo enfoque identificado como amplo, polêmico, dinâmico e ainda está em fase de construção. Em Psicologia, o estudo do fenômeno da resiliência é relativamente recente, no entanto, segundo Souza e Cerveny (2006), a quantidade de artigos publicados sobre o assunto vem crescendo expressivamente, quase que triplicando a cada período de cinco anos.

Sua definição não é clara, tampouco precisa quanto na Física ou na Engenharia, e nem poderia sê-lo, haja vista a complexidade e multiplicidade de fatores e variáveis que devem ser levados em conta no estudo dos fenômenos humanos (Yunes, 2003). Rutter (1999) define a resiliência como um fenômeno de superação de estresse e adversidades, não se constituindo em uma característica ou traço individual. Poletto (2007) complementa, referindo ser a capacidade humana de superar adversidades, resultante da interação permanente e do jogo de forças entre os fatores de risco e proteção, possibilitando, assim, o estabelecimento de sua semelhança com a definição de saúde. Pesce e cols. (2005) entendem por resiliência o conjunto de processos sociais e intrapsíquicos que possibilitam o desenvolvimento saudável do indivíduo, mesmo este vivenciando experiências desfavoráveis. De acordo com Leipold e Greve (2009), não é apenas um conjunto de determinadas reações, mas se trata do resultado de uma combinação de respostas individuais realizadas em uma determinada situação social.

A literatura mostra que, inicialmente, a resiliência referia-se a traços de personalidade e que, embora mais tarde o contexto e as redes de relacionamento fossem consideradas fatores protetores, enfocava-se o desenvolvimento individual saudável em condições difíceis no ambiente familiar ou em situações ambientais, tais como guerras, imigração, problemas sociais, entre outras. A trajetória das pesquisas sobre a resiliência é interessante do ponto de vista que segue o desenvolvimento humano no sentido vertical e a orientação indivíduo-mundo externo no sentido transversal, isto é, inicia abordando a criança, o adolescente, o adulto e, por último, o idoso. Da mesma forma, a definição do conceito evolui do indivíduo (traços de personalidade) para a família (construção relacional) e redes sociais mais amplas (visão ecológica) (Souza \& Cerveny, 2006).

Segundo Sapienza e Pedromônico (2005), apesar de existirem inúmeras definições para o termo, normalmente a resiliência é relacionada ao manejo, pelo indivíduo, de recursos pessoais e contextuais. De acordo com Barbosa (2007), o conceito de resiliência passou de uma fase de "qualidades pessoais" até o conceito mais atual de compreendê-la como um atributo da personalidade desenvolvido no contexto psicosociocultural em que as pessoas estão inseridas.

Papalia e Olds (2000) pontuam que alguns fatores de proteção podem contribuir para a resiliência. Normalmente esses fatores estão relacionados à personalidade da criança, à família, às experiências de aprendizagem, à exposição reduzida ao risco e às experiências compensadoras proporcionadas, por exemplo, por um ambiente escolar favorável. Poletto (2007) refere que a escola é um dos microssistemas que pode atuar diretamente na promoção de resiliência ao desenvolver e incentivar as capacidades e potencialidades da criança, especialmente ao melhorar o desenvolvimento de crianças de famílias menos favorecidas. A escola, ainda, pode incentivar atividades extracurriculares especialmente para adolescentes, facilitando sua inserção futura no mercado de trabalho. Mas a escola pode representar, também, como a família e a instituição, em algumas configurações, fator de risco para o desenvolvimento saudável (Poletto \& Koller, 2008).

A resiliência é estabelecida precocemente na infância e baseia-se no diálogo reflexivo, isto é, a pessoa ser confirmada, vista e respeitada pelo que ela é por um outro significativo ou de confiança. A base para a resiliência já existe na infância e tem de ser considerada na construção da resiliência na adolescência (Lindstron, 2001). Os jovens encaminham-se para a idade adulta através de várias arenas sociais: a família, o contexto social e geográfico, o contexto cultural e histórico, os sistemas educacionais e os sistemas de relacionamento com seus pares e ambientes de trabalho. O autor afirma, ainda, que, quanto mais essas arenas são conectadas em suas estrutura e função, mais fácil fica para o adolescente ver a vida como coerente. 
Garcia (2001) refere existir três tipos de resiliência: social, acadêmica e emocional. A social apresenta como fatores protetivos ter um grupo de amigos e o sentimento de pertencimento ao mesmo, relacionamentos íntimos, bom vínculo com a escola, bem como modelos sociais que promovam uma aprendizagem construtiva nas situações familiares e escolares e equilíbrio entre as responsabilidades sociais e as exigências por obter determinados benefícios. A transmissão de valores, assim como as atitudes positivas dos pais sobre a importância da educação para o futuro de seus filhos, também tem papel fundamental no desenvolvimento de crianças resilientes, essas atitudes colaboram para um melhor desempenho escolar, da mesma forma que ajudam a criança a ser mais competente socialmente no futuro. Segundo o autor, quanto à resiliência acadêmica, a escola pode propiciar o aumento e o fortalecimento de habilidades de resolução de problemas e a aprendizagem de novas estratégias, bem como capacitar professores para auxiliar estudantes com dificuldades. O período escolar contribui para diferentes trajetórias do desenvolvimento, tendo impacto sobre as experiências futuras do indivíduo (Medeiros, Loureiro, Linhares, \& Marturano, 2000). A escola tem sido vista como um dos ambientes, por excelência, para haver o enriquecimento da resiliência (Barbosa, 2007).

A resiliência, de acordo com Pesce e cols. (2005), constitui-se de três dimensões: a primeira é denominada de resolução de ações e valores, ou seja, indica ações relacionadas à energia, persistência, disciplina e à concepção de valores que dão sentido à vida, como a amizade, a realização pessoal, a satisfação e significado da vida; a segunda dimensão é a de independência e determinação, ou seja, caracterizada pela capacidade do indivíduo resolver, sozinho, situações difíceis, lidar com várias situações ao mesmo tempo, aceitar as adversidades e aceitar situações que não pode fazer nada para mudar e, a terceira, é a autoconfiança e capacidade de adaptação a situações, em que indica acreditar que pode resolver seus problemas e que os mesmos dependem mais da pessoa do que dos outros, bem como realizar ações contra sua vontade e manutenção do interesse em coisas que considera importantes.

Sapienza e Pedromônico (2005) relacionam risco, proteção e resiliência. As adversidades não costumam estar isoladas, já que fazem parte do contexto social, envolvendo fatores políticos, socioeconômicos, ambientais, culturais, familiares e genéticos. Dessa forma, os fatores de risco, quando em associação interativa, constituem-se em mecanismos de risco, aumentando a probabilidade ou desencadeando um desfecho negativo no desenvolvimento do indivíduo. Quando muitas situações de risco associam-se, elas dificultam o desenvolvimento, a aquisição de habilidades e o desempenho de papéis sociais.

Entretanto, existem os fatores de proteção que são descritos como recursos pessoais ou sociais que atenuam ou neutralizam o impacto do risco. Os fatores protetores podem atuar como um "escudo" para favorecer o desenvolvimento humano. As variáveis suporte social e autoconceito positivo costumam estar correlacionadas, indicando a existência de um mecanismo através do qual múltiplos fatores protetores promovem a resiliência (Sapienza \& Pedromônico, 2005). Segundo as autoras, a possibilidade de enfrentar fatores de risco e de aproveitar os fatores protetores torna o indivíduo resiliente. Na medida em que o resiliente lança mão de seus recursos positivos para enfrentar as adversidades, a resiliência pode ser considerada fator de proteção para a adaptação do indivíduo às exigências cotidianas. Também apresentam uma lista das principais características encontradas em crianças e adolescentes resilientes: bom funcionamento intelectual, sociabilidade e expressão adequada, autoeficácia, autoconfiança e autoestima elevada, talentos e fé. Nos seus contextos, essas crianças e adolescentes contam com práticas parentais competentes, vantagens socioeconômicas e conexões com redes familiares ampliadas e apoiadoras. No contexto extrafamiliar, mantêm vínculos com adultos e organizações pró-sociais e frequentam a escola.

Estudo realizado por Pesce, Assis, Santos, \& Oliveira (2004) sobre a associação da resiliência com eventos de vida adversos e com fatores de proteção em uma amostra 997 de adolescentes escolares com idade entre 12 e 19 anos de uma rede pública de ensino identificou que a resiliência é um processo interativo entre a pessoa e seu meio, considerado como uma variação individual em resposta ao risco. De acordo com os autores, os dados que foram encontrados em seu estudo mostraram que os eventos traumáticos de vida não estão relacionados com características de um indivíduo resiliente. Não se poderia afirmar que ter vivenciado um tipo de evento ou várias experiências negativas contribui para a capacidade de lidar com a adversidade. No entanto, os fatores de proteção tiveram associação com a resiliência, eles atuam como facilitadores no processo individual de perceber e enfrentar o risco.

Segundo Padovani (2006), o indivíduo encontra-se inserido em um contexto dos quais fazem parte as pessoas que com ele interagem, as conexões entre outras pessoas do ambiente, os vínculos e influências diretas e indiretas que ele recebe a partir das mudanças do ambiente e nas pessoas que dele fazem parte. A resiliência surge, então, como um processo construído a partir da interação da pessoa com o meio. Seja qual for o contexto (família, instituição ou escola), este pode configurar-se como risco ou proteção. No entanto, isto dependerá da qualidade das relações, da presença de afetividade e reciprocidade que tais ambientes oportunizarem. Quando houver conexões positivas entre os contextos e/ou dentro deles, certamente haverá possibilidades que acionam processos de resiliência e favorecem a melhoria da qualidade de vida e adaptação/saúde das pessoas e da sociedade (Poletto \& Koller, 2008).

A noção de vulnerabilidade é composta por elementos que agravam a situação de risco ou impedem respostas satisfatórias diante de situações adversas e deve ser compreendida em três planos básicos: o individual, que está relacionado aos comportamentos, porém não reduzidos a uma ação voluntária, mas associados às condições objetivas do meio natural e social e ao grau de consciência sobre tais comportamentos; o segundo plano é o social, ligado ao 
acesso à informação, aos serviços de saúde, condições de bem estar social; e o terceiro está ligado ao institucional, ao desenvolvimento de ações por instituições específicas visando diminuir, ou eliminar os riscos que tornam os indivíduos mais vulneráveis (Padovani, 2006).

Sapienza e Pedromônico (2005) referem que existem períodos de vida em que o indivíduo está mais vulnerável, por exemplo, a adolescência é um deles, pois é uma fase do desenvolvimento em que ocorrem mudanças físicas e psicológicas; é quando o indivíduo começa a tornar-se independente dos pais e dar mais valor aos pares; é também quando o indivíduo quer explorar uma variedade de situações com as quais ele ainda não sabe bem como lidar. Adolescentes são considerados, por alguns estudiosos, como um segmento da população de elevada vulnerabilidade, devido à estrutura social encontrada em países como o Brasil.

Os estudos sobre resiliência iniciaram-se com as pesquisas sobre a superação de enfermidades, posteriormente focalizaram mais a criança pré-escolar e o adolescente. Atualmente se tem clara a preocupação em conhecer como essa criança ou adolescente vence os desafios impostos pela convivência familiar, escolar e urbana (Souza \& Cerveny, 2006).

Pelo exposto, a investigação buscou contribuir para o conhecimento do tema investigando a relação entre variáveis sócio-demográficas, escolares e contribuição da escola para a resiliência de estudantes do Ensino Médio de uma escola pública.

\section{Método}

\section{Participantes}

A amostra, do tipo acidental, foi composta por 140 alunos do Ensino Médio de uma escola pública de uma cidade da região metropolitana de Porto Alegre - RS. A maioria constituiu-se de meninas (55\%), é solteira $(92,9 \%)$ e possuem idade média de 17,9 anos ( $\mathrm{DP}=4,12$ ). Desses, $26,4 \%$ cursa o $1^{\circ}$ ano, $37,1 \%$ o $2^{\circ}$ ano e, $36,4 \%$ o $3^{\circ}$ ano do Ensino Médio. A maior parte dos alunos mora com os pais ou algum familiar $(97,3 \%)$ não desenvolve nenhuma atividade relacionada a trabalho formal $(53,6 \%)$ e referem já terem tido uma experiência de repetência $(55,7 \%)$ em algum momento de sua vida escolar. Quanto à renda familiar, a maioria informa renda superior a três salários mínimos $(72,1 \%)$ e moram em residência própria (76\%).

\section{Instrumento}

Para operacionalizar os objetivos desta investigação utilizou-se dois instrumentos autoaplicáveis: 1) Questionário para levantamento de variáveis sociodemográficos (sexo, idade, estado civil, atividade laboral, renda familiar), escolares (ano atual, repetência) e psicossocial (contribuição da escola para o desenvolvimento pessoa com variação de opções de 1-ótima a 7-péssima) elaborado especificamente para responder aos objetivos do estudo, tendo como base principal o referencial teórico sobre resiliência; 2) Escala de Resiliência de Wagnild e Young (1993) adaptada para o uso no Brasil por Pesce e cols. (2005). A escala avalia a adaptação psicossocial positiva em face de eventos de vida importantes. Possui 25 itens descritos de forma positiva com resposta tipo likert variando de 1 (discordo totalmente) a 7 (concordo totalmente) e subdivide-se em 3 dimensões: Resolução de ações e valores (RAV-14 itens) caracterizada pela realizações de ações relacionadas a energia, persistência, disciplina, concepção de valores que execução de ações voltadas para dar sentido à vida como a amizade, a realização pessoal, a satisfação e significado da vida; Independência e determinação (ID-6 itens) que indica a capacidade de resolver situações difíceis sozinho, lidar com várias situações ao mesmo tempo, aceitar as adversidades e aceitar situações que não pode fazer nada para mudar ; Autoconfiança e capacidade de adaptação a situações (ACAS-5 itens) que revelam a crença de que a pessoa pode resolver seus problemas e que os mesmos dependem mais dela do que dos outros, realizar ações contra sua vontade mantendo o interesse em coisas que considera importante. A escala adaptada apresentou índice de confiabilidade de 0,80 e nesse estudo 0,70 .

\section{Procedimentos}

Para a coleta dos dados, primeiramente foi realizado um contato com a direção da instituição de ensino tendo sido apresentado o objetivo do estudo a fim de obter a autorização e o apoio para a aplicação dos instrumentos. A aplicação ocorreu no mês de outubro de 2008, nos turnos manhã e noite. Foram realizados os procedimentos éticos conforme legislação do Ministério da Saúde (1997), resolução 196 do Conselho Nacional de Saúde (CNS). Foi esclarecido à direção da escola e aos alunos da instituição tratar-se de uma pesquisa sem quaisquer efeitos avaliativos individuais e/ou grupais e que as respostas seriam anônimas e confidenciais. A pesquisa possui aprovação do Comitê de Ética de afiliação das autoras.

O Banco de Dados foi digitado e posteriormente analisado em pacote estatístico. Foram calculadas médias e desvio-padrão das dimensões de Resiliência e realizada análise de correlação de Pearson para as variáveis quantitativas e $t$ de student e Anova para comparação entre grupos de variáveis qualitativas.

\section{Resultados}

Resultados evidenciam média mais elevada na dimensão de Resolução de ações e valores, seguida pela Autoconfiança e capacidade de adaptação a situações. A menor média foi obtida na dimensão de Independência e determinação, considerando uma escala de pontuação de 1 a 7 , conforme Tabela 1. 
Tabela 1. Média e Desvio-Padrão das dimensões de Resiliência.

\begin{tabular}{lcccc}
\hline Fatores & Min & Max & M & DP \\
\hline RAV & 3,50 & 6,79 & 5,87 & 0,51 \\
ID & 2,50 & 6,17 & 4,50 & 0,79 \\
ACAS & 3,00 & 7,00 & 5,63 & 0,76 \\
\hline
\end{tabular}

Nota: RAV: Resolução de ações e valores; ID: Independência e determinação.

ACAS: Autoconfiança e capacidade de adaptação a situações

$\mathrm{Na}$ análise dos resultados da correlação de Pearson, verifica-se que quanto mais elevada a renda familiar, menor a independência e determinação. Quanto maior a percepção de que a escola contribui para o desenvolvimento pessoal, maior é a dimensão de resolução de ações e valores. Não foi identificada associação das variáveis investigadas com a dimensão de autoconfiança e capacidade de adaptação a situações

Verifica-se através das provas t de student e Anova que nenhuma das variáveis qualitativas apresentou relação com as dimensões de resiliência.

Tabela 2. Matriz de correlação de dimensões de Resiliência e variáveis quantitativas.

\begin{tabular}{lccc}
\hline Variáveis & RAV & ID & ACAS \\
\hline Idade & $-0,018$ & 0,113 & $-0,146$ \\
Escolaridade & $-0,091$ & 0,036 & 0,046 \\
Renda familiar & $-0,014$ & $-0,238^{* *}$ & 0,021 \\
Escolaridade mãe & 0,065 & $-0,165$ & 0,014 \\
Escolaridade pai & 0,029 & $-0,043$ & 0,017 \\
Contribuição escola & $-0,188^{*}$ & $-0,139$ & $-0,060$ \\
\hline
\end{tabular}

Nota: ${ }^{*}$ significativo ao nível de $5 \%$

** significativo ao nível de $1 \%$

RAV: Resolução de ações e valores; ID: Independência e determinação

ACAS: Autoconfiança e capacidade de adaptação a situações

\section{Discussão}

O conceito de resiliência vem sendo construído apenas nos dias atuais. Esse campo da ciência foi recentemente inaugurado e, embora muitos autores tenham se dedicado ao estudo dos fatores que levam o sujeito a lidar positivamente com dificuldades, existe um caminho a ser percorrido até que se chegue a hipóteses mais concretas (Pesce e cols., 2005).
O maior índice de resiliência foi identificado na dimensão de Autoconfiança e capacidade de adaptação a situações, em que o indivíduo indica acreditar que pode resolver seus problemas e que os mesmos dependem mais de si que dos outros, bem como pode realizar ações contra sua vontade e manutenção do interesse em coisas que considera importantes. O desenvolvimento de capacidades de resiliência nos sujeitos passa, conforme Tavares (2001), pela mobilização e ativação das suas capacidades de ser, estar, ter, poder e querer, ou seja, pela sua capacidade de autorregulação e autoestima.

Pesce e cols. (2004) referem que adolescentes com maiores níveis de resiliência têm: autoestima mais elevada; maior supervisão familiar; melhor relacionamento com outras pessoas como amigos e professores; maior apoio social, seja ele emocional, afetivo, informacional e de interação positiva. A dimensão de menor pontuação foi a de Independência e determinação, ou seja, caracterizada pela capacidade do sujeito de resolver situações difíceis sozinho, lidar com várias situações ao mesmo tempo, aceitar as adversidades e aceitar situações que não pode fazer nada para mudar.

Os resultados obtidos não indicam diferença significativa entre os sexos, contrariando resultados obtidos por Pesce e cols. (2004) que verificaram que meninas apresentavam níveis maiores de superação das dificuldades que os meninos. O mesmo resultado ocorreu com relação à idade, ano do Ensino Médio, repetência e atuação profissional. Pode-se hipotetizar que variáveis demográficas e a experiência, tanto profissional como de trabalho, não se constituem fatores de resiliência no grupo estudado, provavelmente pelas características da amostra. São jovens que residem com a família, a maior parte somente estuda e estão cursando o nível médio na idade adequada, 15 a 17 anos, segundo refere o PNAD (2007). Estudo realizado por Padovani (2006) verificou que a escolaridade foi o primeiro item que apresentou diferença entre os adolescentes. É possível pensar que, pelo estudo ter sido realizado somente com jovens de Ensino Médio, a vivência pode não se apresentar tão diferenciada. Supõese que eles tenham sido beneficiados pelas vivências que já tiveram no ambiente escolar ao longo do processo de escolarização.

Os resultados obtidos no estudo indicam associação com a renda familiar e a dimensão de Independência e determinação. Quanto mais elevada a renda familiar, menor a independência e determinação. Esse resultado pode estar relacionado ao perfil da amostra investigada. A maior parte dos alunos possui renda familiar superior a 3 salários mínimos e não desenvolve nenhuma atividade relacionada a trabalho formal. Segundo Barlach (2005), a intensidade com que o ambiente torna-se hostil e estéril é que determina a diferença entre a resiliência e outros processos adaptativos. Na mesma linha, Luthar, Cicchetti e Becker (2000) entendem a resiliência como um processo dinâmico de adaptação positiva às adversidades significativas. Desse modo, resiliência consiste num equilíbrio entre a tensão e a habilidade de lutar, além do aprendizado obtido com os sofrimentos. 
Tabela 3. Relação entre dimensões de Resiliência e variáveis qualitativas.

\begin{tabular}{lcccccccccc}
\hline \multicolumn{1}{c}{ Variáveis } & \multicolumn{3}{c}{ RAV } & \multicolumn{3}{c}{ ID } & \multicolumn{5}{c}{ ACAS } \\
\hline Sexo & & & & & & & & & \\
Feminino & 5,92 & 0,52 & 0,221 & 4,54 & 0,82 & 0,414 & 5,69 & 0,80 & 0,361 \\
Masculino & 5,81 & 0,51 & & 4,43 & 0,75 & & 5,57 & 0,72 & \\
Ensino Médio & & & & & & & & & \\
$1^{\circ}$ ano & 5,95 & 0,43 & 0,551 & 4,46 & 0,79 & 0,913 & 5,60 & 0,75 & 0,846 \\
$2^{\circ}$ ano & 5,87 & 0,56 & & 4,49 & 0,80 & & 5,61 & 0,87 & \\
$3^{\circ}$ ano & 5,82 & 0,52 & & 4,53 & 0,79 & & 5,68 & 0,66 & \\
Atuação Profissional & & & & & & & & & \\
Integral & 5,85 & 0,57 & 0,905 & 4,63 & 0,72 & 0,402 & 5,83 & 0,60 & 0,629 \\
Parcial & 5,91 & 0,63 & & 4,52 & 0,95 & & 5,63 & 0,91 & 0,632 \\
Não trabalha & 5,87 & 0,45 & & 4,42 & 0,77 & & 5,52 & 0,78 & \\
Repetência & & & & & & & & & \\
Sim & 5,85 & 0,58 & & 4,60 & 0,83 & & 5,59 & 0,77 & \\
Não & 5,90 & 0,59 & 0,581 & 4,37 & 0,72 & 0,094 & 5,68 & 0,75 & 0,112 \\
\hline
\end{tabular}

Nota: *Diferença significativa ao nível de $5 \%$.

RAV: Resolução de ações e valores; ID: Independência e determinação; ACAS: Autoconfiança e capacidade de adaptação a situações.

Com relação à dimensão de Resolução de ações e valores, verificou-se que, quanto maior a percepção de que a escola contribui para o desenvolvimento pessoal, maior é o sentimento de energia, persistência e disciplina para agir sobre os problemas e possuir uma concepção de valores que dão sentido à vida, como a amizade, a realização pessoal, a satisfação e o significado da vida. O resultado indica a importância da escola na transmissão de valores e no desenvolvimento de comportamentos úteis para o enfrentamento e resolução de dificuldades. Segundo Poletto (2007), a escola contribui na promoção de processos de resiliência através de projetos e atividades que estimulem as potencialidades individuais e a cooperação. Poletto e Koller (2008) referem que é no contexto escolar que as crianças experienciam inúmeras situações: relações entre pares, grupos, amizade, competição, rivalidade, aprendizagem e descoberta do novo, entre tantas outras.

É importante destacar a não associação da contribuição da escola nas outras dimensões da resiliência, Independência e determinação e Autoconfiança e capacidade de adaptação a situações. Poletto (2007) refere que o discurso da escola, muitas vezes, não parece ter relação com a realidade do cotidiano de alguns jovens. Professoras falam em valores (bem/mal, honesto/desonesto) que têm um significado relativo e circunstancial para eles. São feitas exigências de hábitos de higiene, linguagem, postura e obediência à autoridade discrepantes das condições estruturais e culturais vividas. No entanto, é possível apostar que essas crianças e jovens poderiam se adaptar se algumas de suas expectativas fossem atendidas pela escola.

Amparo, Galvão, Cardinas, \& Koller (2008) destaca que a escola constitui-se numa importante rede de proteção, com a qual os jovens apresentam, em geral, atitudes positivas de confiança e interesse. A continuidade da formação é valorizada e a escola está incluída em projetos futuros destes jovens. Os amigos, professores e pais representam importantes fatores de proteção no contexto escolar.

Não foi identificada associação das variáveis investigadas com a dimensão de Autoconfiança e capacidade de adaptação a situações. Este resultado pode estar relacionado à seleção das variáveis investigadas. De acordo com Pesce e cols. (2004), há fatores socioeconômicos, familiares, escolares e religiosos envolvidos no construto e também um conjunto de fatores inerentes ao indivíduo que contribuem para a força necessária aos jovens que sofrem com a adversidade biológica, física, social ou cognitiva. 


\section{Conclusão}

A escola pode ou não colaborar no desenvolvimento da resiliência, isso depende das interações que ocorrem nessa instituição e do que é construído nessa relação indivíduosociedade. No entanto, para que o indivíduo consiga chegar à escola e ter sucesso, dando continuidade aos seus estudos, é imprescindível que tenha uma família, uma instituição ou uma rede de apoio que o ajude a se manter motivado e com suas necessidades afetivas, sociais e cognitivas atendidas.

Os resultados apontam para a necessidade das escolas atuarem como contextos de valorização para o desenvolvimento dos jovens, principalmente na questão de resolução de ações e construção de valores. Dessa forma, poderão vir a exercer sua cidadania dentro de uma visão ética de mundo. A escola é uma instituição fundamental no processo de formação cidadã e, juntamente com a família, ajuda a promover a construção de sujeitos conscientes, capazes de reconhecer seus direitos e corresponder com seus deveres à sociedade que os integra. A escola pode favorecer o reconhecimento das dificuldades pessoais e propiciar uma atuação para o enfrentamento das limitações. A escola promoverá resiliência se apresentar experiências como desafios e não como ameaças, construindo interações de qualidade com estabilidade e coesão, compondo uma rede de apoio com o ambiente, que demonstre reconhecimento, aceitação e ofereça limites (Amparo e cols., 2008).

É necessário destacar que a literatura sobre o tema no Brasil ainda é incipiente, dificultando a comparação com outros estudos empíricos nacionais. Esta situação é importante, uma vez que hoje já se tem clara a influência de aspectos culturais e contextuais sobre os resultados da resiliência. Deve-se ter cautela com relação aos resultados obtidos neste estudo, uma vez que estes são decorrentes de uma amostra não probabilística, não sendo, portanto, passíveis de generalizações. Sugere-se novos estudos com amostras representativas com uma maior amplitude de variáveis. A realização de pesquisas sobre mecanismos de proteção e resiliência em pessoas expostas a ambientes de risco pode contribuir no esclarecimento de como funcionam esses mecanismos, bem como desvendar a forma como atuam as competências individuais e sociais. Os resultados possibilitam pensar em intervenções voltadas à redução de problemas de desenvolvimento e à promoção da resiliência.

Se a vida é resolver problemas e se os problemas são, na sua maioria, imprevisíveis, é necessário o desenvolvimento da flexibilidade adaptativa. Isto é o que fizeram nossos ancestrais em seu caminho evolutivo (Leipold \& Greve, 2009).

\section{Referências}

Amparo, D. M., Galvão, A. C. T., Cardinas, C., \& Koller, S. H. (2008). A escola e as perspectivas educacionais de jovens em situação de risco. Revista Psicologia Escolar e Educacional, 12(1), 69-88.
Aznar-Farias, M., \& Oliveira-Monteiro, N. R. de. (2006). Reflexões sobre pró-socialidade, resiliência e psicologia positiva. Revista Brasileira de Terapia Cognitiva, 2(2), 39-46.

Barlach, L. (2005). O que é resiliência humana? Uma contribuição para a construção do conceito. Dissertação de Mestrado, Instituto de Psicologia, Universidade de São Paulo, São Paulo.

Barbosa, G. S. (2007). Resiliência? O que é isso? Revista de Divulgação Científica da ECA/USP - São Paulo, 7(39). Recuperado: Jan. 2009. Disponível: <http://www.eca.usp.br/njr/voxscientiae/george_ barbosa_38.htm>

Garcia, I. (2001). Vulnerabilidade e resiliência. Adolescência Latinoamericana, 2(3), 128-130.

Leipold, B., \& Greve, W. (2009). Resilience. A conceptual bridge between coping and development. European Psychologist, 14(1), 40-50.

Lindstron, B. (2001). O significado de resiliência. Adolescência Latinoamericana, 2(3), 133-137.

Luthar, S. S., Cicchetti, D., \& Becker, B.. (2000). The construct of resilience: a critical evaluation and guidelines for future work. Child Development, 71(3), 543-562.

Medeiros, P. C., Loureiro, S. R., Linhares, M. B. M., \& Marturano, E. M. (2000). Autoeficácia e os aspectos comportamentais de crianças com dificuldades aprendizagem. Psicologia: Reflexão e Crítica, 13(3), 327-336.

Padovani, A. S. (2006). Diferenças entre iguais: um estudo sobre características de resiliência entre adolescentes comunitários e privados de liberdade. Recuperado: 10 nov. 2008. Disponível em: $<$ http://www.bvs-psi.org.br/tcc/201.pdf>

Papalia, D. E., \& Olds, S. W. (2000). Desenvolvimento humano. Porto Alegre: Artes Médicas.

Pesce, R. P., Assis, S. G., Avanci, J. Q., Santos, J. C., Malaquias, J. V., \& Carvalhaes, R. (2005). Adaptação transcultural, confiabilidade e validade da escala de resiliência. Cadernos de Saúde Pública, 21(2), 436-448.

Pesce, R. P., Assis, S. G., Santos, N., Oliveira, R. de V. C. (2004). Risco e proteção: em busca de um equilíbrio promotor de resiliência. Psicologia: Teoria e Pesquisa, 20(2), 135-143.

Poletto, M. (2007). Contextos ecológicos de promoção de resiliência para jovens em situação de risco. Dissertação de Mestrado, Universidade Federal do Rio Grande do Sul, Porto Alegre.

Poletto, M., \& Koller, S. H. (2008). Contextos ecológicos: promotores de resiliência, fatores de risco e de proteção. Estudos de Psicologia - Campinas, 25(3), 405-416. 
Rutter, M. (1999) Resilience concepts and findings: implications for family therapy. Journal of Family Therapy, 21, 119-144.

Sapienza, G., \& Pedromônico, M. R. M. (2005). Risco, proteção e resiliência no desenvolvimento da criança e do adolescente. Psicologia em Estudo, 10(2), 209-216.

Síntese de Indicadores Sociais 2008 com base na Pesquisa Nacional por Amostra de Domicílios. (Pnad, 2007). Recuperado: 15 set. 2008. Disponível: <http://www.ibge.gov.br/home/presidencia/noticias/ noticia_visualiza.php?id_noticia $=1233 \& i d$ pagina $=1>$
Souza, M. T. S. de, \& Cerveny, C. M. de O. (2006). Resiliência Psicológica: Revisão da Literatura e Análise da Produção Científica. Revista Interamericana de Psicologia, 40(1) 119-126.

Tavares, J. (2001). A resiliência na sociedade emergente. Em J. Tavares (Org.), Resiliência e educação (pp.43-76). São Paulo: Cortez.

Yunes, M. A. M. (2003). Psicologia positiva e resiliência: o foco no indivíduo e na família. Psicologia em Estudo, 8(número especial), 75-84.

Recebido em: 10/09/2009

Reformulado em: 10/06/2010

Aprovado em: 11/06/2010

\section{Sobre as autoras}

Lidiane Peltz (lidianepeltz@yahoo.com.br)

Universidade Luterana do Brasil, Canoas - RS

Maria da Graça Moraes (psimoraes@terra.com.br)

Universidade Luterana do Brasil, Canoas - RS

Mary Sandra Carlotto (mscarlotto@gmail.com)

Universidade Luterana do Brasil, Canoas - RS

\section{Correspondência}

Lidiane Peltz,

Rua: Pinheiro Machado n. ${ }^{\circ} 2614$, apto 26, Bairro São Pelegrino

Caxias do Sul- RS, CEP 95020172

\section{Agradecimento}

Ao CNPq. pela bolsa produtividade para Mary Sandra Carlotto 